\title{
Mass yield, isotopic yield and kinetic energy distributions for heavy masses in thermal neutron induced fission
}

\author{
A. Bail ${ }^{1, a}$, O. Serot ${ }^{1}$, H. Faust ${ }^{2}$, U. Koester ${ }^{2}$, T. Materna ${ }^{2}$, I. AlMahamid ${ }^{3}$, and T.E. Kuzmina ${ }^{4}$ \\ 1 CEA Cadarache, DEN/DER/SPRC/LEPh, 13108 Saint-Paul-lez-Durance, France \\ 2 Institut Laue-Langevin, 38042 Grenoble, France \\ 3 Wadsworth Center, New-York State Department of Health, Albany NY 12201, USA \\ 4 V.G. Khlopin Radium Institute, 194021 Saint Petersburg, Russia
}

\begin{abstract}
A more accurate knowledge of heavy fission product yields is needed to improve our understanding of the fission process and to increase the efficiency in nuclear reactor operation. High resolution measurements of fission observables can be done with the recoil mass spectrometer Lohengrin at the Institut Laue-Langevin in Grenoble, France, which was designed to measure fission fragment characteristics from neutron induced fission. The mass separator is situated at the research reactor of the institute and permits the placement of an actinide layer in a high thermal neutron flux. It separates fragments according to atomic mass, kinetic energy and ionic charge state by the action of magnetic and electric fields, and allows to determine these distributions, isotopic yields, and the fragment gamma-decay characteristics. Almost all fissile isotopes rangings from Th to Cf have been investigated with Lohengrin, and in particular mass and nuclear charge distributions for light fission products have been determined. The experimental set-up used to investigate the mass yield and the fission fragment kinetic energy distributions in the heavy mass region, or in the light mass region, is done by coupling a high resolution ionization chamber to the spectrometer. For low mass fission products a double anode ionization chamber is used to determine furthermore isotopic yields within a mass line. For fission fragments of higher mass this separation is no longer possible, and gamma-spectrometry is used instead to identify the contributing nuclear charges. The experimental set-up is shortly described in the present paper, and we present preliminary results for measurements of thermal neutron induced fission in ${ }^{235} \mathrm{U}\left(\mathrm{n}_{\text {th }}, \mathrm{f}\right),{ }^{239} \mathrm{Pu}\left(\mathrm{n}_{\text {th }}, \mathrm{f}\right)$ and ${ }^{241} \mathrm{Pu}\left(\mathrm{n}_{\text {th }}, \mathrm{f}\right)$.
\end{abstract}

\section{Introduction}

Even if nuclear fission was discovered almost seventy years ago, complementary data are still needed to improve our understanding of the process. Data already listed in data bases are not always very accurate, and the list of the possible reactions is not exhaustive. Moreover, considerable differences can be found between the various evaluated data libraries (fig. 1), in particular between ENDF, JEFF and JENDL evaluations. To improve the efficiency of nuclear reactor operation, mass yield of fission products effecting reactivity need to be known with better accuracy, in particular for the minor actinides. Likewise the fission models need more and better nuclear data to be compared with [1].

For systems which undergo fission in a thermal neutron flux, detailed results for kinetic energy, nuclear mass, and nuclear charge have been measured on Lohengrin, in particular ${ }^{229} \mathrm{Th},{ }^{233} \mathrm{U},{ }^{235} \mathrm{U},{ }^{238} \mathrm{~Np},{ }^{239} \mathrm{~Np},{ }^{241} \mathrm{Pu},{ }^{245} \mathrm{Cm}$ and ${ }^{249} \mathrm{Cf}[2]-$ [8]. However, only the light fission yields for these actinides have been investigated. We present here a study for fission product characteristics in the heavy mass region.

\section{The Lohengrin mass spectrometer}

The Lohengrin recoil-mass spectrometer is a nuclear facility which uses low-energy fission reactions for fragment production (fig. 2). It allows us to study fragment distributions from

\footnotetext{
${ }^{a}$ Presenting author, e-mail: bail@ill.fr
}

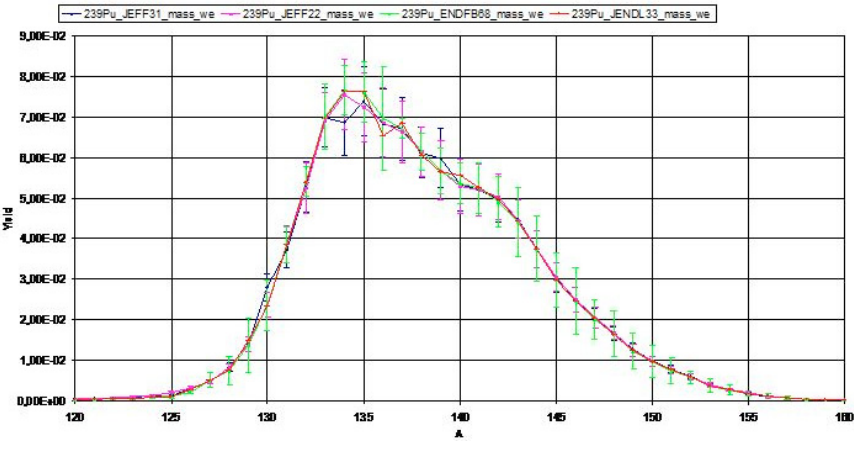

Fig. 1. Heavy mass yields for the ${ }^{239} \mathrm{Pu}\left(\mathrm{n}_{\mathrm{th}}, \mathrm{f}\right)$ reaction from the main evaluated data libraries.

thermal neutron induced fission with a very high resolution. The beam intensity at the separator allows also detection of gamma-rays, conversion electrons, beta-rays, delayed neutrons, and coincidences between these particles.

The Lohengrin fission source is typically a thin layer of a fissile material placed close to the core of ILL's research reactor, in a high thermal-neutron flux of $5.3 \cdot 10^{14}$ neutron $/ \mathrm{cm}^{2} / \mathrm{s}$.

Fission products emerging from the target are selected by a combination of a magnetic and an electric sector field, whose deflections are perpendicular to each other. Both sector fields have focussing properties in their plane of deflection. In agrement with Bohr's theorem high energetic fission product are leaving the target with high ionic charge states. The combined action of the two fields separates ions with the same velocity 


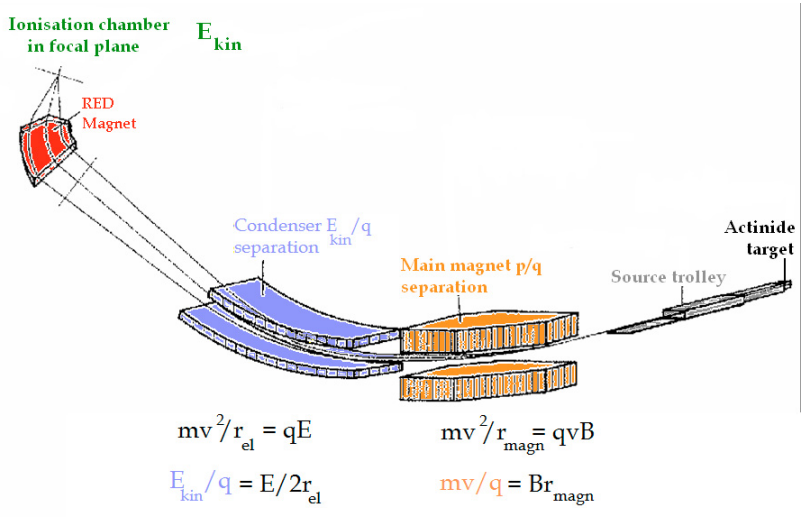

Fig. 2. Schematic view of the Lohengrin mass spectrometer.

$$
\text { Pt-wire (anode) }
$$

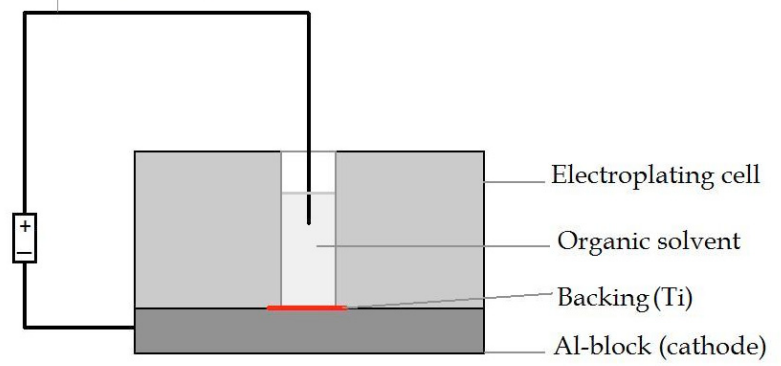

Fig. 3. Schematic view of the cell used for electrodeposition.

into different parabolas at the exit slit of the spectrometer, according to their $\mathrm{A} / \mathrm{q}$ and $\mathrm{E}_{k} / \mathrm{q}$ values. Here $\mathrm{A}, \mathrm{q}$ and $\mathrm{E}_{k}$ are mass, ionic charge state and kinetic energy of the ions, respectively. The energy dispersion in the direction along each parabola amounts to $7.2 \mathrm{~cm}$ for $1 \%$ difference in energy, and the mass dispersion perpendicular to each parabola amounts to $3.24 \mathrm{~cm}$ for $1 \%$ mass difference. In recent years a focusing magnet has been installed at the exit slit at the spectrometer, which increases the particle density by a factor of 7 . The flightpath for the fission products is $23 \mathrm{~m}$, and the separation time is of the order of $2 \mu \mathrm{sec}$, so that fission products reach the detector before undergoing beta-decay.

For spectroscopy work on neutron rich nuclei different equipments may be installed next to the exit slit of Lohengrin such as ionization chambers, surface barrier detectors, Si and Ge detectors, plastic scintillators and long counters for delayed neutrons. Also a fast tape transport is available for different experiments.

\section{Target production}

In the past fissile targets were obtained from the Euratom facilities in Geel, from the Radium Institute of St. Petersburg and from Arzamas, Russia, as well as from differents laboratories in the USA. At the ILL we produce targets of ${ }^{235} \mathrm{U}$ with

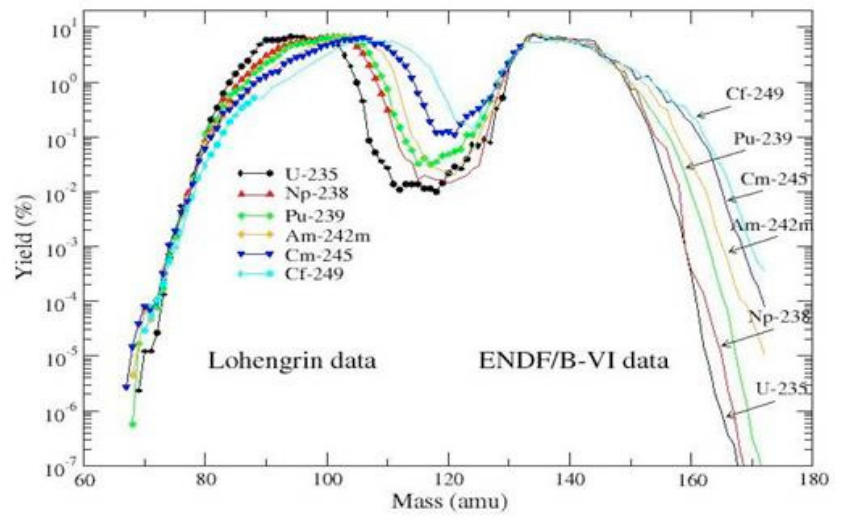

Fig. 4. Absolute mass yields from the neutron-induced fission of different nuclei: data from the mass separator Lohengrin (connected symbols) completed with the ENDF/B-VI nuclear-library data (lines), figure taken from [12].

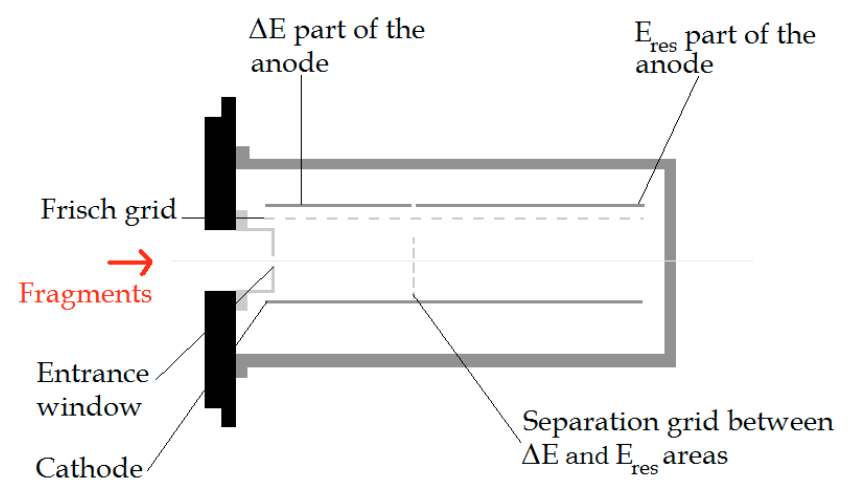

Fig. 5. Schematic view of the ionization chamber.

different thicknesses and sizes. We have initiated two different procedures to make the targets:

1. by electrodeposition

2. by painting.

\subsection{Preparation of targets by electro-deposition}

The electro-deposition technique is well suited for the preparation of actinide targets on metallic backing materials, with deposition yields approaching 100\% [9]. For electro-deposition, the actinide is dissolved in nitric acid and mixed with isopropanol. By applying an electric current during electrolysis no dissociation of the uranyl complex occurs, and probably the same compound as originally dissolved is deposed. Figure 3 shows a schematic view of the cell used for molecular electrodeposition. An electroplating cell made out of teflon confines the area to be plated, and acts as a solution container. The backing foil with an active target area of $7 \mathrm{~cm}^{2}$ is pre-mounted into an aluminium block, which serves as the cathode. A platinum wire is used as anode material. Electrodeposition is carried out by applying a voltage from $200 \mathrm{~V}$ to $600 \mathrm{~V}$. 


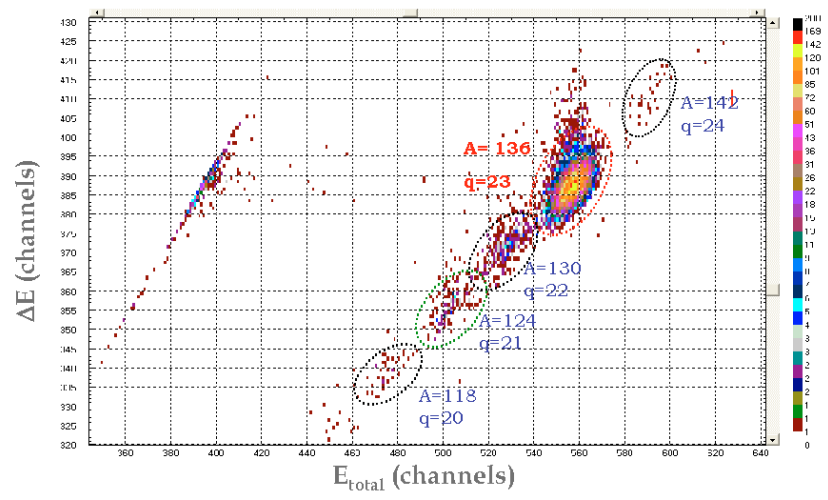

Fig. 6. Example of acquisition for the mass yields of ${ }^{239} \mathrm{Pu}\left(\mathrm{n}_{\mathrm{th}}, \mathrm{f}\right)$ measurement.

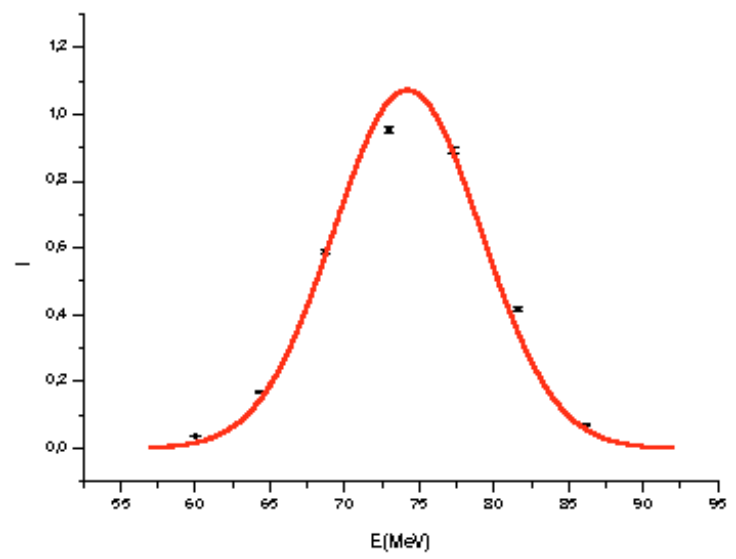

Fig. 7. $\mathrm{E}_{k}$-distribution for the ionic charge state $\mathrm{q}=22$ for the nuclear mass $A=134$ of ${ }^{235} U\left(n_{\text {th }}, f\right)$.

\subsection{Preparation of targets by painting}

The solution used in the painting process is similar to the one used for preparations of targets by electro-deposition, i.e., the nitrate of the actinide dissolved in isopropanol. The painting is done with a wide brush, and the way of painting chosen is parallel strokes [11]. The deposit of the actinide material is done by alternating painting and heating to $600^{\circ} \mathrm{C}$ in order to decompose and evaporate the organic binder.

With the use of both fabrication techniques a large amount of different targets with thicknesses between $50 \mu \mathrm{g} / \mathrm{cm}^{2}$ and $600 \mu \mathrm{g} / \mathrm{cm}^{2}$ on titanium backing were produced with good homogeneity.

\section{Mass yields and kinetic energy distributions}

As we can see in the figure 4 thermal fission mass yields of many compound systems have been measured with Lohengrin in the light fission products region, but there is none in the heavy product region.

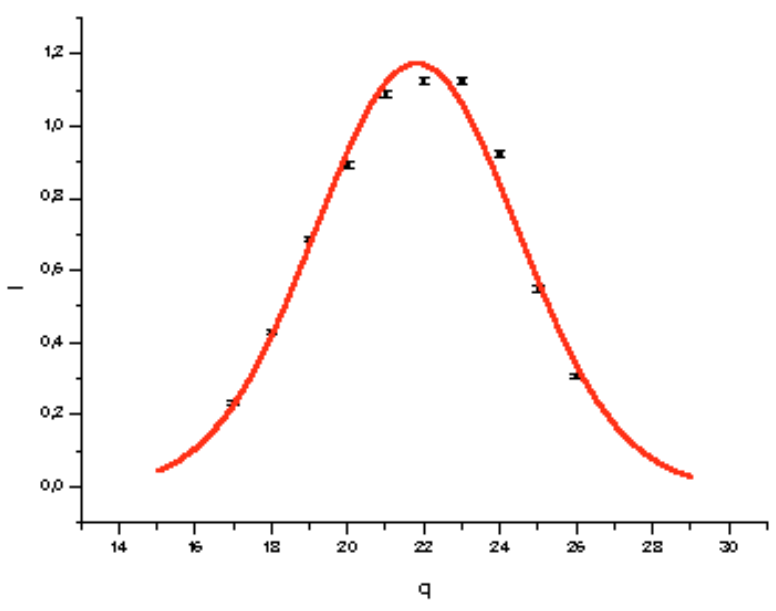

Fig. 8. q-distribution for the kinetic energy $\mathrm{E}_{k}=61 \mathrm{MeV}$ for the nuclear mass $A=134$ of ${ }^{235} U\left(n_{t h}, f\right)$.

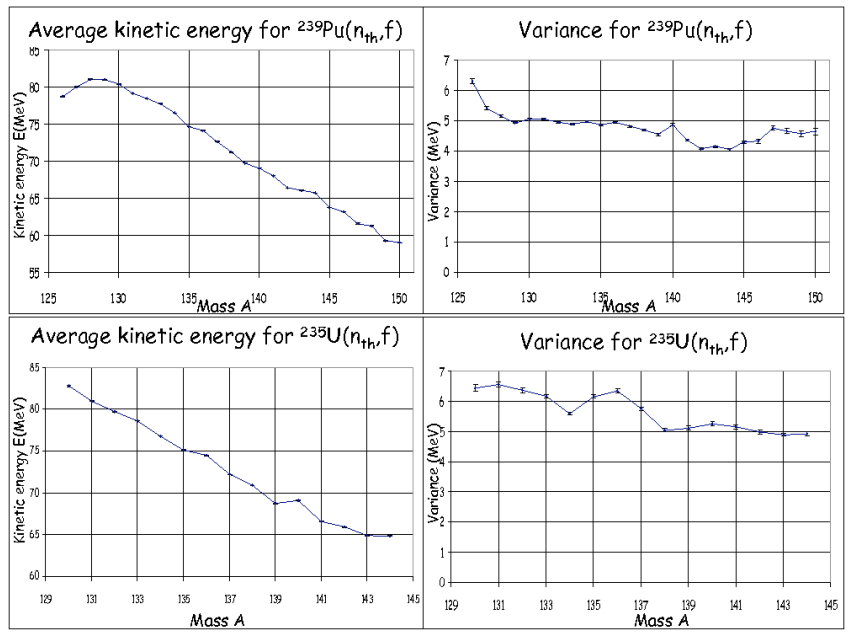

Fig. 9. Mean kinetic energy (top left) and variance (top right) from fission of ${ }^{239} \mathrm{Pu}\left(\mathrm{n}_{\mathrm{th}}, \mathrm{f}\right)$ and mean kinetic energy (bottom left) and variance (bottom right) from fission of ${ }^{235} \mathrm{U}\left(\mathrm{n}_{\mathrm{th}}, \mathrm{f}\right)$.

The aim of the present experiment was the determination of yield and kinetic energy for heavy fission fragments. We used an ionization chamber at the exit slit of the spectrometer which allows to determine the kinetic energy $\left(\mathrm{E}_{k}\right)$ value of the separated fragments, giving thus the mass (A) and ionic charge state $(q)$ values.

The ionization chamber is filled with isobutane at $\mathrm{P} \simeq$ 40 mbar pressure. The high voltage applied between the anode and the Frisch grid is $400 \mathrm{~V}$, and the same voltage is applied between the Frisch grid and the cathode (fig. 5).

This set-up allows us to obtain the complete mass yields of an isotope by integration over kinetic energy and ionic charge distributions for every mass (figs. 6-8). Last year we investigated two fission systems:

1. some measurements have been done for the determination of mass yield in ${ }^{235} \mathrm{U}\left(\mathrm{n}_{\mathrm{th}}, \mathrm{f}\right)$ in order to validate the method; 2. the heavy mass yield for ${ }^{239} \mathrm{Pu}\left(\mathrm{n}_{\mathrm{th}}, \mathrm{f}\right)$ was investigated.

Up to now the results of the energy distributions are evaluated (fig. 9). Mean kinetic energies and variances as function of 


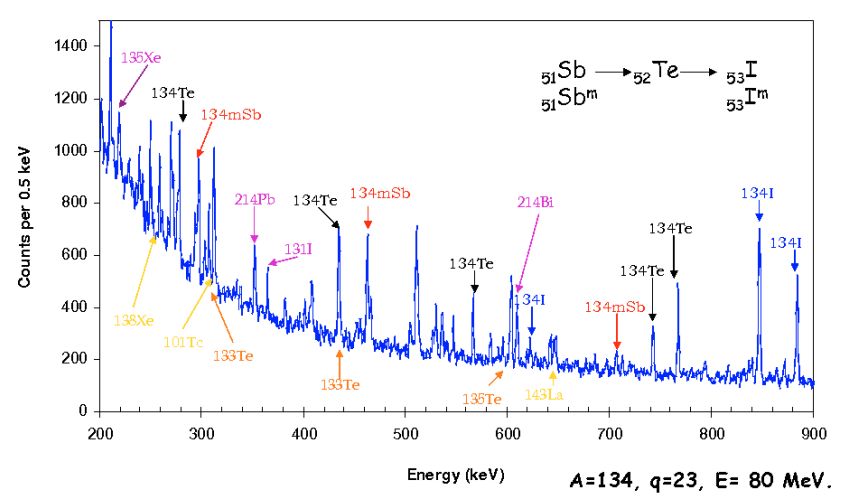

Fig. 10. Example of gamma-spectrum for isotopic yields determination from ${ }^{241} \mathrm{Pu}\left(\mathrm{n}_{\mathrm{th}}, \mathrm{f}\right)$.

mass number show the expected behavior as deduced for a binary reaction on the basis of energy and momentum conservation laws.

\section{Isotopic yields}

For low mass fission products with $\mathrm{Z} \leq 42$ the isotopic components within one mass line is determined by inspection of the ionization track in a split anode ionization chamber. This method is no longer applicable for fragments with nuclear charges $\mathrm{Z}$ higher than 42 . Therefore we tried to determine isotopic yield by gamma-spectrometry. As the beta-decays of fission products are often followed by gamma de-excitation, and because these decays occur after their fligh path in the spectrometer, beta-gamma concidences can be used to determine isotopic yields. For this purpose the fission products are implanted in a moving tape which is coupled to the ionization chamber at the focus position of the spectrometer. Two germanium clover detectors are used to measure the gamma-decay with high efficiency, whereas the beta de-integration process is detected by a proportional gas counter or a scintillator. An example of a gamma-spectrum obtained for mass $\mathrm{A}=134$ is given in figure 10. The different isotopes contributing for this mass are $\mathrm{Sb}$, Te and I, and their gamma-decay charasteristic are clearly visible. We can also see the contaminant contributions of the present measurement: ${ }^{133} \mathrm{Te}$ and ${ }^{135} \mathrm{Te}$; of the long-life isotopes of the previous measurement: ${ }^{101} \mathrm{Tc},{ }^{138} \mathrm{Xe}$ and ${ }^{143} \mathrm{La}$; and of the background: ${ }^{131} \mathrm{I},{ }^{214} \mathrm{~Pb}$ and ${ }^{214} \mathrm{Bi}$. From the respective strenghts of the gamma transitions, the isotopic yields will be determined.

\section{Conclusion and perspectives}

Experiments on fission products in the heavy wing from ${ }^{235} \mathrm{U}\left(\mathrm{n}_{\text {th }}, \mathrm{f}\right)$ and ${ }^{239} \mathrm{Pu}\left(\mathrm{n}_{\mathrm{th}}, \mathrm{f}\right)$ were performed on the mass separator Lohengrin to determine characteristics of kinetic energy, mass yield and isotopic yield. Kinetic energy distributions for ${ }^{235} \mathrm{U}\left(\mathrm{n}_{\mathrm{th}}, \mathrm{f}\right)$ and ${ }^{239} \mathrm{Pu}\left(\mathrm{n}_{\mathrm{th}}, \mathrm{f}\right)$ have been evaluated and data treatment for mass and isotopic yield are in progress.

\section{References}

1. H. Goutte et al., Low energy fission: Dynamics and scission configurations, AIP Conf. Proc. 798, 76 (2005).

2. M. Djebara et al., Nucl. Phys. A 425, 120 (1984).

3. U. Quade et al., Nucl. Phys. A 487, 1 (1988).

4. W. Lang et al., Nucl. Phys. A 345, 34 (1980).

5. I. Tsekhanovich et al., Nucl. Phys. A 688, 633 (2001).

6. D. Rochman et al., Nucl. Phys. A 710, 3 (2002).

7. T. Friedrichs et al., Investigations of mass, charge, and energy of thermal neutron induced fission of ${ }^{245} \mathrm{Cm}$ and ${ }^{241} \mathrm{Pu}$, in Proceedings of the Second International Workshop on Nuclear Fission and Fission-Product Spectroscopy, AIP Conference Proceedings 447, Seyssins, France, p. 231.

8. M. Djebara et al., Nucl. Phys. A 496, 346 (1989).

9. K. Eberhardt et al., Nucl. Instrum. Meth. A 521, 208 (2004).

10. W. Parker, Nucl. Instrum. Meth. 16, 355 (1962).

11. L.V. Drapchinsky et al., Nucl. Instrum. Meth. A 438, 116 (1999).

12. http://www.ill.fr/nfp/npp/Pn1/Physics.htm. 